\title{
Revistas jurídicas e interdisciplinariedad: la evaluación a debate*
}

\author{
LUIS ANTONIO SOBRADO GONZÁLEZ * \\ HUGO PICADO LEÓN***
}

\begin{abstract}
RESUMEN: A partir de la experiencia de la Revista de Derecho Electoral, editada por el Tribunal Supremo de Elecciones de Costa Rica desde enero de 2006, este artículo somete a debate los criterios ausentes en los procesos de evaluación y de edición de las revistas jurídicas. El carácter interdisciplinario, innovador y dinámico de los estudios electorales y su diálogo permanente con el régimen jurídico constituyen un desafío para algunos moldes tradicionales de las revistas jurídicas. Se plantea como pregunta de investigación: ¿qué criterios editoriales y de evaluación ha debido contemplar una revista dedicada al derecho electoral para no renunciar a nutrirse y a dialogar con aproximaciones extrajurídicas de lo electoral? El artículo se organiza en tres partes: la primera describe las principales características de la Revista de Derecho Electoral, en la segunda se explica el carácter interdisciplinario de los estudios electorales y su relación con el derecho electoral; finalmente, se analizan los impactos y los retos que de esa interdisciplinariedad se derivan para la política editorial y para los criterios de evaluación de una revista dedicada al derecho electoral.
\end{abstract}

PALABRAS CLAVE: publicaciones académicas, revista electrónica, derecho electoral, interdisciplinariedad.

ABSTRACT: Based on the experience of the Electoral Law Review, edited by the Supreme Court of Elections of Costa Rica since January 2006, this article discusses the criteria that are absent in the evaluation and editing processes of legal reviews. The interdisciplinary, innovative and dynamic nature of electoral studies and their permanent dialogue with the legal regime, constitutes a challenge for some traditional molds of legal magazines. It is posed as a research question: what editorial and evaluation criteria should a journal devoted to electoral law have to contemplate in order not to give up nourishing itself and dialoguing with extra-legal approaches to the electoral? The article is organized in three parts: the first describes the main characteristics of the Electoral Law Review; in the second, the interdisciplinary nature of electoral studies and its relationship with electoral law are explained; Finally, the impacts and challenges derived from this interdisciplinarity for editorial policy and for the evaluation criteria of a magazine dedicated to electoral law are analyzed.

KEY WORDS: academic publications, electronic journal, electoral law, interdisciplinarity.

\footnotetext{
* Artículo de investigación Número Especial de Aniversario. Recibido el 17 de abril de 2020 y aceptado para su publicación el 2 de septiembre de 2020.

** Magistrado Presidente en el Tribunal Supremo de Elecciones, Costa Rica. Profesor y Coordinador en la Universidad de Costa Rica. / Isobrado@tse.go.cr / orcid.org/0000-0002-4840-9628

*** Magistrado Suplente en el Tribunal Supremo de Elecciones, Costa Rica. / hpicado@tse.go.cr / orcid.org/ 0000-0001-57247387
} 


\section{Introducción}

Parece natural que las revistas jurídicas evalúen los artículos a partir de métodos de investigación y razonamientos propios del derecho. Los trabajos sobre análisis normativo vinculados al deber ser, los de descripción o valoración jurisprudencial o las propuestas de reforma normativa se bastan con las aproximaciones y recursos propios de la investigación jurídica y su retórica.

Sin embargo, existen ramas del derecho cuya materia exige un abierto intercambio con disciplinas ajenas a lo jurídico. El derecho electoral es uno de esos casos. Este artículo somete a debate los criterios ausentes en los procesos de evaluación y de edición de las revistas jurídicas, con base en la experiencia acumulada durante los quince años de existencia de la Revista de Derecho Electoral (RDE). El carácter interdisciplinario, innovador y dinámico de los estudios electorales y su diálogo permanente con el régimen jurídico constituye un desafío para algunos moldes tradicionales de las revistas jurídicas.

Se plantea como pregunta de investigación: ¿cuáles criterios editoriales y de evaluación ha debido contemplar una revista dedicada al derecho electoral para no renunciar a nutrirse y a dialogar con aproximaciones extrajurídicas de lo electoral? El artículo concluye que una revista jurídica dedicada a una rama cuyo objeto exija un intercambio con áreas de especialización distintas al derecho debe incorporar a profesionales con distintas formaciones capaces de valorar adecuadamente el rigor metodológico y la consistencia de los aportes que se someten a su consideración.

El artículo se organiza en tres partes: la primera describe las principales características de la RDE, en la segunda se explica el carácter interdisciplinario de los estudios electorales y su relación con el derecho electoral; finalmente, se analizan los impactos y los retos que de esa interdisciplinariedad se derivan para la política editorial y para los criterios de evaluación de una revista dedicada al derecho electoral.

\section{La Revista de Derecho Electoral}

La RDE es una publicación electrónica semestral editada por el Tribunal Supremo de Elecciones de Costa Rica (TSE) desde enero de 2006. Surgió con la intención de llenar un acusado vacío en el estudio del derecho electoral costarricense, hasta aquel momento lánguido en cuanto a análisis doctrinal. A pesar de la sólida institucionalidad electoral derivada de la Constitución Política de 1949, todavía a inicios del siglo XXI la investigación sobre el derecho electoral costarricense se encontraba en pañales. Si acaso cabría citar la monografía de Rubén Hernández Valle (1990) y una decena de artículos regados en revistas jurídicas y obras colectivas. ${ }^{1}$

\footnotetext{
${ }^{1}$ Sobre la configuración del régimen electoral costarricense en la Constitución de 1949, véase: Sobrado González, 2010.
} 
Mediante el acuerdo n. ${ }^{\circ}$ TSE105-2005 del 27 de octubre de 2005, el TSE dispuso la creación de la RDE como publicación electrónica, de periodicidad semestral, que se colocaría en la página web del organismo electoral bajo la filosofía de acceso abierto (Open Access), por lo que sus contenidos están disponibles en internet bajo formato accesible y gratuito. EI TSE procuró incentivar y divulgar las investigaciones de académicos y funcionarios electorales, nacionales y extranjeros. ${ }^{2}$

La RDE fue concebida como "un espacio académico para la publicación y gestión de nuevo conocimiento en estudios electorales nacionales e internacionales. Con una perspectiva interdisciplinaria en el abordaje de los temas y dirigida a público académico y ciudadanía en general, coadyuva a la reflexión sobre las instituciones básicas para la convivencia política democrática". ${ }^{3}$

Quince años después, la RDE no solo ha llenado un vacío doctrinal, sino que se ha convertido en una publicación referente de los estudios electorales en Costa Rica, además de albergar un significativo acervo de investigaciones sobre los sistemas electorales latinoamericanos y del mundo. Como ejemplo de su peso, cabe señalar que el Programa del Curso Derecho Constitucional II, de la Facultad de Derecho de la Universidad de Costa Rica incluye como lecturas obligatorias un total de 23 trabajos publicados en la RDE. ${ }^{4}$

La revista cuenta con cuatro secciones habituales que albergan artículos de investigación sobre la materia electoral: la sección "Autores invitados", que recoge aportes de investigadores invitados por la revista; la sección "Aportes desde el TSE", que reúne artículos elaborados por funcionarios del TSE; la sección "Colaboraciones externas", que publica los artículos remitidos por autores ajenos al TEE; y la "Sección especial", que se publica ocasionalmente, cuando se logra reunir un conjunto de artículos relativos a un tema común de vigente interés. Hasta el primer semestre de 2020 se han publicado 29 números, con un total de 352 artículos, para un promedio que supera los 12 artículos por número (ver tabla 1).

Tabla 1. Secciones y número de artículos publicados en la RDE.

\begin{tabular}{cccccc}
$\begin{array}{c}\text { RDE } \\
\text { número }\end{array}$ & $\begin{array}{c}\text { Autores } \\
\text { invitados }\end{array}$ & $\begin{array}{c}\text { Aportes } \\
\text { desde el } \\
\text { TSE }\end{array}$ & $\begin{array}{c}\text { Colaboraciones } \\
\text { externas }\end{array}$ & $\begin{array}{c}\text { Sección } \\
\text { especial }\end{array}$ & $\begin{array}{c}\text { Total de } \\
\text { artículos }\end{array}$ \\
\cline { 2 - 3 } 1 & 1 & 4 & 1 & 0 & 6 \\
2 & 2 & 0 & 5 & 0 & 7 \\
3 & 2 & 3 & 0 & 3 & 8 \\
4 & 1 & 2 & 2 & 5 & 10 \\
5 & 1 & 3 & 4 & 2 & 10 \\
6 & 1 & 2 & 2 & 4 & 9 \\
7 & 1 & 4 & 2 & 3 & 10
\end{tabular}

\footnotetext{
2 Sobre la creación de la RDE, véase: Mena Hidalgo y Montero Solano, 2012.

${ }^{3}$ Véase: https://www.tse.go.cr/revista/objetivo.htm. Consultado el 14 de abril de 2020.

${ }^{4}$ Cabe señalar que ese curso se dedica al estudio del derecho electoral. Sobre la enseñanza del derecho electoral en Costa Rica, véase: Sobrado González y Brenes Villalobos, 2016.
} 


\begin{tabular}{cccccc}
8 & 1 & 5 & 5 & 0 & 11 \\
9 & 1 & 0 & 0 & 10 & 11 \\
10 & 1 & 4 & 8 & 0 & 13 \\
11 & 1 & 2 & 5 & 2 & 10 \\
12 & 1 & 1 & 3 & 6 & 11 \\
13 & 1 & 3 & 1 & 9 & 14 \\
14 & 2 & 3 & 0 & 10 & 15 \\
15 & 1 & 3 & 5 & 4 & 13 \\
16 & 2 & 4 & 3 & 3 & 12 \\
17 & 1 & 4 & 5 & 4 & 14 \\
18 & 1 & 2 & 8 & 5 & 16 \\
19 & 2 & 3 & 5 & 6 & 16 \\
20 & 12 & 0 & 0 & 0 & 12 \\
21 & 2 & 3 & 6 & 0 & 11 \\
22 & 2 & 2 & 5 & 4 & 13 \\
23 & 1 & 2 & 7 & 3 & 13 \\
24 & 1 & 3 & 0 & 12 \\
25 & 1 & 0 & 7 & 12 & 20 \\
26 & 1 & 5 & 5 & 0 & 11 \\
27 & 2 & 5 & 5 & 3 & 15 \\
28 & 1 & 2 & 6 & 105 & 13 \\
29 & 1 & 1 & 113 & 352 \\
\hline Total & 47 & 74 & 0 & & \\
\hline
\end{tabular}

Fuente: Elaboración propia.

Con base en la información generada por la herramienta Google Analytics, que provee datos a partir de 2012, la línea de tendencia de acceso a la RDE es ascendente, pues las visitas han venido aumentando desde las 17,657 en 2012 hasta 81,835 en 2019 (ver tabla 2). Llaman la atención los incrementos en visitas de los años 2016 y 2018, pues prácticamente duplican las del año precedente: en 2015 se contabilizan 35,728 visitas, mientras que en 2016 el número de visitas se incrementó a 69,620; por su parte, en 2017 el número de visitas fue de 54,866, mientras que en 2018 el número de visitas fue de 106,692. Ese comportamiento resulta coincidente con los ciclos electorales, pues en 2016 se llevaron a cabo elecciones municipales y en 2018 hubo comicios presidenciales y legislativos.

Tabla 2. Visitas a RDE 2012-2019. 


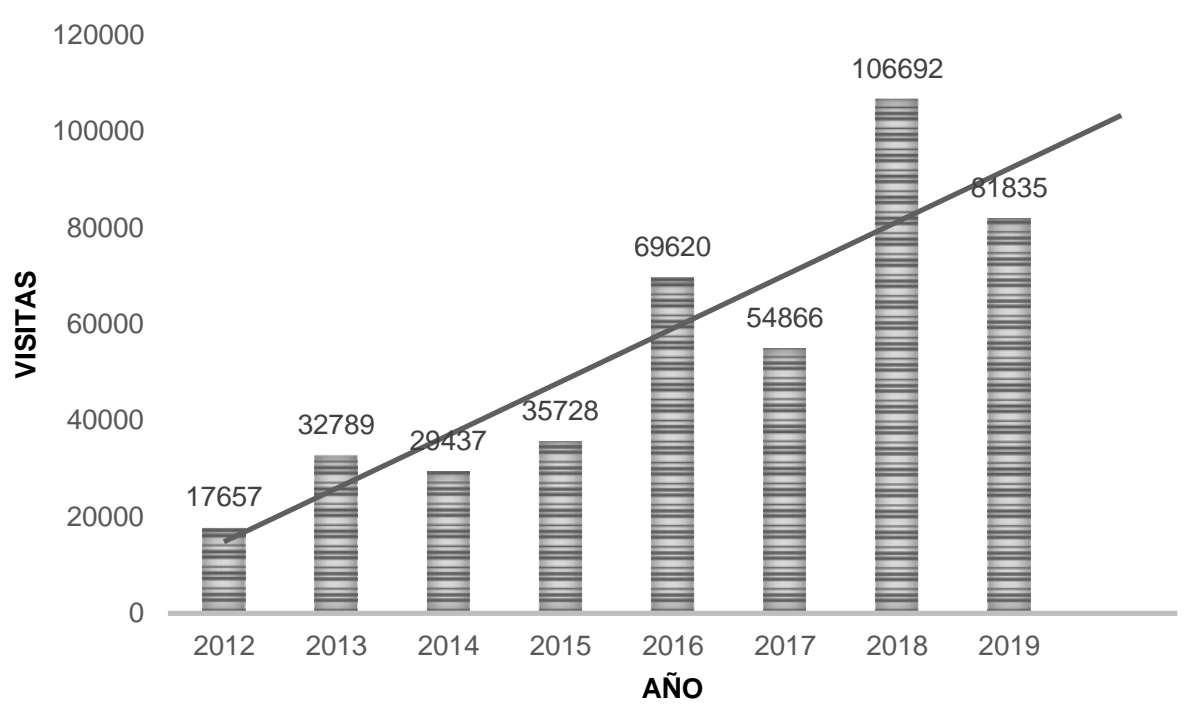

Fuente: Centro de documentación IFED, con base en Google Analytics.

La revista no tiene formato de magazín informativo, sino de revista científica, por lo cual todos sus artículos deben cumplir estrictos criterios preestablecidos de forma y de fondo, que son evaluados por un consejo editorial bajo el proceso de revisión por pares de tipo "doble ciego".

Por su apego a esos parámetros, la RDE ha logrado permanecer indexada en Dialnet, en Latindex, en la Red Iberoamericana de Innovación y Conocimiento Científico (REDIB), en la Plataforma de Publicaciones Digitales ISSUU y en el Directory of Open Access Journals (DOAJ).

La logística propia para la edición, el montaje y la publicación de la RDE se coordina a través del Instituto de Formación y Estudios en Democracia (IFED), unidad administrativa que concentra las funciones pedagógicas y académicas del TSE. Eso permite que la RDE mantenga actualidad, pues frecuentemente los artículos publicados provienen de las actividades académicas organizadas por el IFED, sean conversatorios, seminarios, conferencias o mesas redondas. Además, el IFED asegura un soporte técnico y administrativo necesario para la RDE.

\section{El carácter interdisciplinario de los estudios electorales}

La RDE, tal como su nombre lo indica, inicialmente fue concebida como una revista de carácter primordialmente jurídico. Sin abandonar ese rasgo, la naturaleza interdisciplinaria de los estudios electorales condujo paulatinamente a su dirección y a su consejo editorial a adoptar una perspectiva más amplia que facilitara el diálogo entre el derecho y las demás disciplinas que se ocupan de lo electoral, habida cuenta de que el derecho electoral se nutre de otras áreas de conocimiento extrajurídicas. 
Existe una relación simbiótica entre el derecho electoral y las distintas aproximaciones al fenómeno comicial desde la sociología, las ciencias políticas, las ciencias de la comunicación colectiva, el mercadeo, la psicología, la administración pública, la ingeniería industrial, la informática, la historia y la economía, entre otros campos de investigación. La creación, la interpretación y la aplicación del conjunto de normas jurídicas vinculadas al sufragio constituyen el eje del derecho electoral. Sin embargo, los procesos mediante los cuales se promulgan, se interpretan, se integran, se aplican, se derogan o se anulan normas interactúan con realidades políticas, económicas y sociales que trascienden lo normativo. ${ }^{5}$

El derecho electoral se ha beneficiado de los avances en la comprensión de lo electoral provenientes de otras disciplinas académicas. Solo por citar algunos ejemplos: a) desde Duverger hasta Lijphart, las ciencias políticas lograron identificar los elementos de los sistemas electorales y medir sus efectos; b) desde Ostrogorski y Michels hasta Cox, la estasiología lleva más de un siglo estudiando los efectos de las reglas electorales sobre los sistemas de partidos y viceversa; c) una de las tradiciones más fructíferas en el estudio de lo electoral proviene de las ciencias políticas comparadas, sobre todo a partir de la denominada Tercera Ola de la Democratización; d) los estudios sobre comportamiento electoral se han nutrido de disciplinas como la estadística o la economía; e) las campañas electorales han sido terreno fértil para trabajos desde la sociología, la psicología social, el marketing y las ciencias de la comunicación colectiva; y f) la influencia de la informática es cada vez más importante en la gestión comicial y continuamente plantea nuevos desafíos a las reglas sobre administración electoral y sobre campañas electorales.

Lo electoral se explica a partir de sistemas políticos reales y complejos. Por esa razón, el derecho electoral debe evitar la endogamia, para lo cual es necesario dialogar con otros enfoques y tradiciones heurísticas que se han dedicado a lo electoral. Esto equivale a ir más allá del análisis del derecho positivo y del tradicional estudio formalista. El derecho electoral surge y opera en contextos dinámicos de permanente lucha por el poder político en el que interactúan electores, partidos políticos, candidatos, gobiernos, parlamentos, sindicatos, grupos de presión, medios de comunicación y actores externos.

Por otra parte, el derecho electoral se encuentra íntimamente vinculado a categorías o conceptos que, en sí mismos, son objeto de interés para diversas disciplinas. La democracia, en muchas de sus acepciones (desde las ciencias sociales empíricas hasta la filosofía política), suele estar vinculada al sufragio. Autores como Schumpeter, Dalh o Sartori no conciben una democracia sin elecciones, pues el ejercicio del sufragio universal, libre, transparente y periódico se entiende como condición necesaria (aunque no suficiente) para la democracia.

De lo anterior se colige la necesaria amplitud y adaptabilidad de las revistas dedicadas al derecho electoral. Al fin y al cabo, el derecho electoral no solamente

\footnotetext{
${ }^{5}$ Sobre el carácter interdisciplinario del derecho electoral, véase Picado León, 2008, pp. 5-7.
} 
constituye una ramificación del derecho público, sino que también es parte de una más heterogénea familia de estudios sobre democracia y elecciones.

\section{Evaluación de artículos y políticas editoriales}

Debido al carácter heterogéneo de los estudios electorales, la RDE acoge trabajos con enfoques interdisciplinarios que complementan las aproximaciones jurídicas. Cuando un texto se dedica al análisis del comportamiento electoral utilizando técnicas estadísticas, investiga las transformaciones en el sistema de partidos tomando como variable independiente los clivajes sociales o estudia el discurso político de los candidatos mediante herramientas informáticas para el análisis de texto. Estamos en presencia de trabajos vinculados al sufragio y al derecho electoral, que eventualmente pueden aportar insumos para la creación, la interpretación o la modificación de normas. Sin embargo, esos textos no pueden ser valorados desde una perspectiva exclusivamente jurídica.

En estos casos, el trabajo editorial se complica. La evaluación de los artículos requiere ir más allá de la constatación del cumplimiento de los requisitos formales del texto, como pueden ser el número de palabras, la correcta citación de fuentes o la estructura y redacción del trabajo. Aparte de lo formal, el valor de una investigación reside en su aporte al conocimiento teórico o empírico del problema propuesto, así como en la correcta utilización de los métodos y de las estrategias de investigación.

En los procesos de evaluación y de edición de una revista jurídica cuyo objeto se nutre de otras ramas del conocimiento, es necesario mantener un delicado equilibrio epistemológico. Por una parte, se requiere cierta flexibilidad para acoger metodologías y enfoques de investigación propias de las diversas ramas de la investigación social; por otra, es fundamental mantener el rigor propio del conocimiento científico, no especulativo. Una revista demasiado flexible perderá seriedad científica; una revista excesivamente rigurosa perderá información valiosa.

Para lograr ese equilibrio el proceso editorial requiere una visión amplia, jurídica pero también interdisciplinaria, que le permita valorar con precisión la validez, la pertinencia y la corrección metodológica de los trabajos que, más allá de lo exclusivamente normativo, aprovechen avances de otros espacios de conocimiento.

Con ese objeto, la RDE se fue adaptando paulatinamente. Actualmente, la RDE cuenta con un consejo editorial integrado por funcionarios del TSE y con un consejo científico externo. Ambos consejos están integrados por profesionales en diferentes disciplinas que tienen en común su interés por lo electoral. Además de abogados, entre los miembros del consejo editorial y del consejo científico externo se encuentran profesionales en sociología, ciencias políticas, pedagogía, relaciones internacionales, economía o bibliotecología. 
En la tarea concreta de revisar y evaluar los aportes enviados a consideración del consejo editorial, este suele requerir el apoyo de otros funcionarios del TSE con formación en filología, historia, psicología, estadística, administración y comunicación, entre otras áreas de especialización.

Paralelamente, el formulario utilizado para evaluar los artículos se ha adaptado de manera que permita tanto la valoración de textos de análisis jurídico como de aquellos que incorporen perspectivas propias de otras disciplinas.

\section{Conclusión}

El derecho electoral dialoga continuamente con disciplinas no jurídicas. Lo electoral se explica a partir de sistemas políticos reales y complejos. Esos fenómenos han sido estudiados desde hace muchas décadas por la sociología, las ciencias políticas, la economía y la psicología social, entre otras ramas del conocimiento.

De lo anterior se colige la necesaria amplitud y adaptabilidad de las revistas dedicadas al derecho electoral. Al fin y al cabo, el derecho electoral no solamente es una ramificación del derecho público, sino que también es parte de la más heterogénea familia de los estudios sobre democracia y elecciones.

Una revista jurídica dedicada a una rama cuyo objeto exija un intercambio con áreas de especialización distintas al derecho debe incorporar a profesionales con distintas formaciones, capaces de valorar adecuadamente el rigor metodológico y la consistencia de los artículos que se les someten a evaluación.

\section{Bibliografía}

Hernández Valle, Rubén, Derecho electoral costarricense, San José, Juricentro, 1990.

Mena Hidalgo, María Mayela y Montero Solano, Rocío, "Estudio bibliométrico de la Revista de Derecho Electoral del Tribunal Supremo de Elecciones", Revista de Derecho Electoral (Costa Rica), núm. 14, julio-diciembre 2012 (consultado el 26 de marzo de 2020). Disponible en: https://www.tse.go.cr/revista/art/14/mena montero.pdf.

Picado León, Hugo, "Implicaciones jurídicas del sistema electoral", Revista de Derecho Electoral (Costa Rica), núm. 6, julio-diciembre 2008 (consultado el 26 de marzo de 2020). Disponible en: https://www.tse.go.cr/revista/art/6/PICADO.pdf.

Sobrado González, Luis Antonio, "El régimen electoral en la Constitución de 1949", Revista de Derecho Electoral (Costa Rica), núm. 9, enero-junio 2010 (consultado el 26 de marzo de 2020). Disponible en: https://www.tse.go.cr/revista/art/9/sobrado gonzalez(1).pdf.

Sobrado González, Luis Antonio y Brenes Villalobos, Luis Diego, "Enseñanza del derecho electoral en Costa Rica", Revista de Derecho Electoral (Costa Rica), 
núm. 22, julio-diciembre 2016 (consultado el 26 de marzo de 2020). Disponible en: https://www.tse.go.cr/revista/art/22/sobrado brenes.pdf.

Universidad de Costa Rica, Facultad de Derecho, Programa del Curso DE-3004

Derecho Constitucional II, 2018 (consultado el 26 de marzo de 2020). Disponible

en:

https://repositorio.so.ucr.ac.cr/programas ciencias sociales/Derecho/2018/iiciclo/DE3004\%20-\%20Derecho\%20Constitucional\%20II.pdf. 